\title{
Powerful Problems for Powerful Qualities
}

\author{
Henry Taylor $^{1}$ iD
}

Received: 25 April 2019 / Accepted: 26 November 2019 / Published online: 24 December 2019

(c) The Author(s) 2019

\begin{abstract}
The powerful qualities view of properties is currently enjoying a surge in popularity. Recently, I have argued that the standard version of the view (associated with C.B. Martin and John Heil) is no different from a rival view: the pure powers position. I have also argued that the canonical version of the powerful qualities view faces the same problem as the pure powers view: the dreaded regress objection. Joaquim Giannotti disagrees. First, Giannotti thinks that the standard version of the powerful qualities view can be differentiated from the pure powers view. Second, Giannotti argues that the powerful qualities view is not susceptible to the regress objection. Third, he argues that there is another reasonable version of the powerful qualities view available, which makes use of the notion of 'aspects'. In this note, I respond to Giannotti. I argue that all three of Giannotti's arguments are unsuccessful.
\end{abstract}

\section{Powerful Qualities}

The powerful qualities view attempts to steer a middle path between categoricalism [the view that properties are not essentially dispositional, but are 'categorical' or 'qualitative' (Armstrong 1997)] and the pure powers view [that all properties have de re dispositional essences and that there is nothing more to their nature than that (Bird 2007)]..$^{1}$ The powerful qualities view claims that properties are both qualitative and dispositional (Martin 2008; Heil 2003; Taylor 2013; Jacobs 2011; Mørch 2018). The most popular version of the powerful qualities view is the identity theory, on which the dispositionality and qualitativity of a property are identical to each other, and to the property itself (Heil 2003, p. 111; Martin 2008, p. 63).

\footnotetext{
${ }^{1}$ Having a dispositional essence is something a property has tout court, not something it has relative to a description. Thus, if property $\mathrm{A}$ has a dispositional essence, and $\mathrm{A}=\mathrm{B}$, then $\mathrm{B}$ has a dispositional essence as well.

Henry Taylor

j.h.taylor.1@bham.ac.uk

1 Department of Philosophy, ERI Building, University of Birmingham, Birmingham B15 2TT, UK
} 
I have recently argued that the identity theory and the pure powers view collapse into the same view (Taylor 2018). Call this the collapse argument. In the same paper, I also argued that the dreaded regress problem for the pure powers view also applies to the identity theory. Call this the regress argument. Giannotti defends the identity theory from both the collapse and the regress arguments (forthcoming). ${ }^{2}$ In this paper, I argue that these defences are unsuccessful (Sects. 2, 3). I then examine an alternative (non-identity) version of the powerful qualities view, which Giannotti suggests (Sect. 4). In Sect. 5, I briefly outline some positive directions for the powerful qualities view.

\section{The Collapse Argument}

The collapse argument runs as follows (for full details, see Taylor 2018). The supposed difference between the identity theory and the pure powers view lies in the fact that the former claims that properties are qualities, whilst the latter claims that properties are 'purely' powerful. However (goes the argument) we need to disambiguate between various interpretations of what a 'quality' is. Once we do this, we see that for every proposed interpretation of 'quality', either both identity theorists and pure powers theorists are committed to the claim that properties are 'qualities', or both of them are committed to rejecting the claim that properties are qualities. Thus, advocates of both views accept that properties are qualities in some senses, and not qualities in others.

Previously, I have considered a range of different interpretations of 'quality' in the course of the collapse argument (2018, pp. 1425-1430). I will not list them here, as this paper's focus is Giannotti's interpretation. Giannotti says 'the powerful qualities theorist conceives of qualities as actual properties of their bearers' (Giannotti Sect. 1). He says 'to be a quality is to be an actual property of a bearer' (Giannotti Sect. 1). Giannotti also quotes Heil, saying that qualities are 'here and now, actual, not merely potential features of the objects of which they are qualities' (Heil 2012, p. 59, Giannotti Sect. 1). On this conception, to be a quality is to be an actual, not merely potential, feature of an object. Since this is Giannotti's conception of a quality, this is the conception I will adopt in this paper.

Note how thin this notion of a 'quality' is. On this view, being qualitative amounts to nothing more than being an actual, real, 'here and now' feature of a bearer. Giannotti himself says that a 'consequence of this conception is that every actual property is a quality' (Giannotti Sect. 1). Clearly the pure powers theorist would agree that properties are qualities in this sense. Pure powers theorists accept that properties are powers, and also that they are actual, real, not merely potential, features of objects (Bird 2007, pp. 102-103).

Giannotti resists the collapse argument in the following way. He accepts that pure powers theorists and identity theorists both claim that properties are qualitative, on the thin meaning of 'quality' that he is working with (Giannotti Sect. 1). He then

\footnotetext{
2 All references to Giannotti are to this paper. Since page numbers are not available for this paper yet, all references are to section number, not page number.
} 
invokes the notion of a dual nature. He says: "It is the "dual nature" of a powerful quality that makes it dispositional and qualitative' (Giannotti Sect. 1). He then claims that, conversely, pure powers have a 'powerful but not qualitative nature' (Giannotti Sect. 1). Finally, he claims that a pure power 'has qualitativity only derivatively, in virtue of its dispositional nature' (Giannotti Sect. 1).

We can extract three core ideas from these quotations:

(i) Powerful qualities are dispositional and qualitative because they have a dual nature.

(ii) Pure powers, by contrast, have a powerful but not a qualitative nature.

(iii) Pure powers have qualitativity only derivatively, in virtue of their dispositionality.

Start with (i). What does it mean to have a 'dual nature'? In the context of the identity theory, it cannot mean that properties have distinct qualitative and dispositional elements, because to the identity theorist, the dispositional and the qualitative are identical. So what does it amount to? In explication of the notion of a dual nature, Giannotti cites C. B. Martin. In the passage that Giannotti cites, Martin says:

I take properties to have a dual nature: in virtue of possessing a property, an object possesses both a particular dispositionality and a particular qualitative character (2008, p. 44).

In this quotation, to have a dual nature means that a property contributes to an object's dispositionality and its qualitative character. Recall that, on the notion of 'qualitative' that Giannotti is working with, for a property to be qualitative means that it is an actual, real feature of the object that instantiates it. So, inserting this notion of the 'qualitative' into the understanding of 'dual nature' from Martin gives us the result that, for a property to have a dual nature means that it contributes both to an object's dispositionality, and to its actual, real features. But if that's what 'dual nature' means, then pure powers theorists also claim that properties have a dual nature. They claim that properties contribute to the dispositions of objects that instantiate them, and to the way that the object actually, really is. Thus, both identity theorists and pure powers theorists accept (i). We cannot distinguish the views here.

Turn to (ii). On the thin meaning of 'qualitative' that we are working with, (ii) would interpret pure powers theorists as claiming that properties have a powerful nature, but that this nature is not an actual, real feature of the object that instantiates it. This is hard to understand. How could a property have a powerful nature (or any nature, for that matter) that is not an actual, real feature of the objects that instantiate it? In any case, this isn't the view that pure powers theorists have in mind. They think that the dispositional nature of a property is a real and actual feature of the object (Bird 2007). Thus, both identity theorists and pure powers theorists would reject (ii). Again, we can't distinguish the views in this way. ${ }^{3}$

\footnotetext{
3 There is another possible interpretation of (ii) (thanks to an anonymous referee for suggesting this). On this interpretation, properties have a dispositional nature, and are also qualitative. However, given that the property has a dispositional nature, the contribution that the property makes to the object's disposi-
} 
Finally, point (iii): Giannotti claims that a pure power only has qualitativity derivatively, in virtue of its dispositional nature. But this isn't an accurate representation of the pure powers position. Pure powers theorists do not claim that the qualitativity (which here, recall, just means the contribution that a property makes to the actual, real features of an object) of a property arises in virtue of its dispositionality in any way. It is not as though the dispositionality of a property somehow produces the property's actuality and reality. Rather, to the pure powers theorist, the dispositionality of a property is an actual real feature of the object that instantiates it (e.g. Bird 2007, pp. 101-102). ${ }^{4}$ So none of these three claims can distinguish the views.

Giannotti helpfully distinguishes between three versions of the identity theory. Giannotti does not use these distinctions to resist the collapse argument, so I mention them only briefly. On one version, the qualitative and the dispositional are different concepts for a property (Giannotti Sect. 3). On the second, the qualitative and the dispositional are co-referential descriptions of a property. For the third, the qualitative and the dispositional are aspects of a property, and there is no real distinction between these aspects. The points about the collapse argument made just now apply to all of these. Both the pure powers theorist and the identity theorist will accept that there are a variety of concepts and descriptions that we can use to think about and refer to properties (Taylor 2017, 2018, pp. 1428-1429). With regard to the third version of identity theory, the points made above still apply. The pure powers theorist claims that the dispositionality of a property is an actual, real feature of the object that instantiates it. So, if we want to refer to these as dispositional and qualitative 'aspects', then the pure powers theorist will claim that there is no real distinction between these two aspects. Again, there's no distinction to be drawn here.

\section{The Regress Argument}

I now turn to the claim that the identity theory is also susceptible to the same dreaded vicious regress that threatens the pure powers view. Of course, if we accept the collapse argument, then this regress problem will follow immediately. They're the same view, so they face the same problems. However, it is worth noting that the regress argument can be stated even without assuming the truth of the collapse

\footnotetext{
Footnote 3 (continued)

tional profile is modally fixed (the same property makes the same dispositional contribution in all modal contexts). Conversely, the qualitative contribution that the property makes is modally variable (properties can make different qualitative contributions in different modal contexts). This interpretation of the pure powers view faces difficulties. It implies that the qualitative and dispositional contributions that a property makes to an object are different (because they have different modal profiles), whereas advocates of the pure powers view will claim that they are the same (that is, the dispositional contributions a property makes are real, actual features of the object). The view also faces a problem of explanatory impotence. If the (dispositional) nature of a property does not fix the qualitative contribution that the property makes to an object in a modally strict way, then what does? The view has no resources to answer this question.

4 Similar things will go for the claim that a property's qualitativity is grounded in its dispositionality (thanks to an anonymous referee for suggesting this). The canonical pure powers view isn't that a property's actual, real contribution to an object is grounded in its dispositionality. It is that the property's dispositionality is itself an actual real contribution to the object in question.
} 
argument. I first summarise the reasons to think that the identity theory faces the regress (for full details, see Taylor 2018, pp. 1435-1438). Then I examine Giannotti's argument against this, and respond to it. To be clear: my claim is not that the regress is fatal to either view. My claim is that it is as much of a problem for identity theory as it is for the pure powers view (Taylor 2018, p. 1436).

As most thinkers (including Giannotti and myself) see it, the most threatening version of the regress for the pure powers view comes from E. J. Lowe. It goes as follows. The pure powers view is committed to the claim that the dispositional nature of a property is sufficient to fix its identity: to individuate it, to make it that which it is and not another distinct thing. But the dispositionality of a property is itself individuated by other properties. Specifically, it is individuated by the properties that have stimulus and manifestation relations to the property. ${ }^{5}$ On the pure powers theory, these properties are themselves dispositional, and so their identity is fixed by stimulus/manifestation relations to other properties, and so on... We end up with either a vicious circle or a vicious regress, with the result that no property ever gets its identity fixed (Lowe 2006, p. 138).

The regress problem arises because the pure powers view is committed to the claim that dispositionality is sufficient to fix the identity of a property. This is problematic because (so goes the argument) dispositionality cannot be sufficient to fix the identity of a property on pain of vicious regress. How does this bear on the identity theory? The identity theorist asserts a threefold identity: the property, the dispositionality and the qualitativity are all identical. Crucially, since the dispositionality of a property is identical with the property, the identity theorist is committed to the claim that the dispositionality of a property must be sufficient to fix the identity of the property. This is the very claim that the regress targets. So, the identity theorist is committed to exactly the same claim that makes the pure powers view susceptible to the regress. The problem thus applies in the same way: the dispositionality cannot be sufficient to fix a property's identity on pain of vicious regress.

Obviously, the identity theorist will respond by pointing out that they take properties to be qualities. Thus, the identity theorist can claim that the qualitative nature of a property individuates it. Setting aside some of the details (Taylor 2018, pp. 1435-1438), the basic problem with this response is that the regress objection has the conclusion that nothing with a dispositional nature can be sufficient to fix the identity of a property. On the identity theory, we cannot respond to this objection by deferring to qualities, because the very same problem arises here. On the identity theory, a quality does have a dispositional nature, because it just is a dispositionality (it's identical to one). If nothing with a dispositional nature can fix the identity of a property, then a quality cannot do so either (because a quality is itself just a dispositionality, on this view).

Put simply, if we are sure that a dispositionality is insufficient to do the individuating work, then we cannot solve the problem simply by renaming the dispositionality using the name 'quality'. To paraphrase Lewis, something cannot

\footnotetext{
5 Martin rejects talk of 'stimuli' and 'manifestations, in favour of 'reciprocal disposition partners' (2008). This subtlety does not matter here.
} 
individuate a property just by being called a 'quality', any more than one can have mighty biceps just by being called 'Armstrong' (1983, p. 366).

My concern is how Giannotti resists the regress argument. He supposes (for argument's sake) that 'what individuates a property must be a qualitative feature of a property in virtue of which it has a certain dispositionality' (Giannotti Sect. 2). He claims that this would be incompatible with the pure powers view, but that the identity theorist can accept it. He then goes on to say that (in order to be compatible with the identity theory) 'such an individuating qualitative nature would be also dispositional' (Giannotti Sect. 2).

From these quotations, we can extract two core claims that make up the view Giannotti suggests for the identity theorist to avoid the regress argument.

1. Properties have a qualitative nature, which individuates the property, and it is in virtue of this that they have a certain dispositionality.

2. This qualitative nature is itself dispositional.

One problem with this approach will now be clear. As Giannotti rightly says, if this picture is to be compatible with the identity theory, then the qualitative nature that individuates the property must be dispositional [point (2)]. The regress objection aims to show that nothing with a dispositional nature can individuate a property, on pain of regress. The individuating feature in the view just proposed is still dispositional, so the regress will recur. In other words, the problem is that the identity theory must accept (2) in order to be consistent (otherwise there would be a non-dispositional element to a property, which the identity theory cannot allow, because it claims that a property is identical with a dispositionality). But (2) will just cause the regress objection to apply, because the regress applies to any view on which something with a dispositional nature is sufficient to individuate a property.

Another issue with the view embodied in (1-2) is Giannotti's claim that a pure powers theorist cannot accept it. In support of this, he says 'If a property has a certain dispositionality in virtue of its qualitative nature, then we must abandon the central tenet of [the pure powers view], namely that a property's nature is dispositional' (Sect. 2). Now, the pure powers view is committed to the claim that a property is individuated by something dispositional (and in this sense, that a property's nature is dispositional). But there is no tension between this claim and the view embodied in (1-2). On the view proposed, what does the individuating work is qualitative (1), but since that quality is itself dispositional (2), it follows that what does the individuating work is itself dispositional. So there is no clash with the pure powers theory here.

The final issue with the view embodied in (1-2) is that it is incompatible with the identity theory. Giannotti suggests that a property is individuated by a qualitative feature, and that the dispositionality of a property arises in virtue of this qualitativity [point (1)]. Call this qualitative feature $Q$, and we can use ' $D_{1}$ ' for the dispositionality that arises in virtue of $\mathrm{Q}$. Consider the implications of this idea. $\mathrm{D}_{1}$ and $\mathrm{Q}$ are not identical because (on this view) $\mathrm{D}_{1}$ arises in virtue of $\mathrm{Q}$, 
and if the 'in virtue of' relation is to be non-trivial, it must be an asymmetric relation. Nothing can bear an asymmetric relation to itself, so it follows that $Q \neq D_{1}$. As Giannotti also says [point (2)], Q is itself dispositional. So there is another dispositionality, $\mathrm{D}_{2}$, such that $\mathrm{Q}=\mathrm{D}_{2}$. We have several elements: $\mathrm{Q}$ is identical with $\mathrm{D}_{2}$, and $\mathrm{D}_{1}$ arises in virtue of that. But on the identity theory, the dispositionality of a property is identical with its qualitativity, and each is identical with the property itself. Clearly that has been violated here, because there is an element of a property $\left(\mathrm{D}_{1}\right)$ which isn't identical with its qualitativity.

\section{The Dual-Aspect Account}

I have argued that Giannotti's attempts to resist the collapse and regress arguments are unsuccessful. However, Giannotti does not only discuss the identity version of the powerful qualities view. He also develops a non-identity version of the view, which he calls the dual-aspect account. This account demands discussion, because if what I have argued concerning the identity view is correct, then powerful qualities theorists will be forced either to explore alternative, non-identity versions of the view, or accept that their view is the same as the pure powers view. They are unlikely to do the latter, because the pure powers view is one that they have always rejected (Heil 2012, p. 76).

The dual-aspect view draws crucially on the notion of aspects of a property. Aspects are 'ways of properties' (Giannotti Sect. 3). Giannotti says that 'To say that a property has certain aspects means that it exists in certain manners or ways' (Giannotti Sect. 3). He sketches out four features of aspects. First that 'the nature of a property determines its aspects' (Giannotti Sect. 3). Second, that 'we can consider an aspect of a property by means of abstraction' (Giannotti Sect. 3). Third that 'the aspects of a property supervene upon it' (Giannotti Sect. 3). Finally, that 'the aspects of a property are modally fixed' (Giannotti Sect. 3).

Giannotti then proposes a version of the powerful qualities view on which the dispositionality and the qualitativity of a property are aspects in the above sense. As Giannotti points out, this is compatible with the claim that these aspects are identical, but he sets this aside, and accepts instead that the dispositional and qualitative aspects are distinct. This is in the interests of developing a version of the powerful qualities view that is not a version of the identity theory (Giannotti Sect. 4). He calls this the dual-aspect account. Importantly, Giannotti does not commit to the dualaspect account, but presents it as one way that the powerful qualities theorist could go, if the identity versions turn out to be untenable (Giannotti Sect. 4). Nonetheless, I take it to be a serious suggestion, and I analyse it as such.

The core problem with the dual-aspect account arises when we put together two of its core commitments. The first commitment is the thin notion of the qualitative that Giannotti is working with. Recall that, on the notion of 'qualitativity' that Giannotti is working with, for something to be qualitative just means that it is an 'actual, here and now, not merely potential' feature of the object that instantiates 
it (Giannotti Sect. 1). The second commitment is that the dispositional aspect of a property is distinct from its qualitative aspect.

To see how these two claims cause trouble, consider that for something to be qualitative just means that it is an actual, real feature of the object that instantiates it. So (on Giannotti's notion of 'qualitative'), to say that a dispositional aspect is distinct from the qualitative aspect is just to say that the dispositional aspect is distinct from the aspect that is an actual, real feature of the object that instantiates it. However, this implies that the dispositionality is not itself an actual, real feature of the object that instantiates the property.

Notice that Giannotti cannot claim that the dispositional aspect of a property is a real, actual feature of the object. This option is not available, because if he does make this claim, then the dispositionality would fulfil the requirements to count as 'qualitative', on his thin notion of the qualitative. If the dispositional aspect of a property fulfils the requirement to count as 'qualitative' then it will no longer be the case that the dispositional aspect of a property is distinct from the qualitative aspect of the property, and the distinctness claim at the heart of the dual-aspect view would collapse.

This criticism is not a flat-footed insistence that dispositionality cannot constitute a genuine addition to the actual, real features of objects (cf. Armstrong 1997, p. 80). ${ }^{6}$ This simple objection has long been known to be question begging, on the grounds that dispositionalities contribute to a property's causal role, which is an actual and real contribution to the object (e.g. Bird 2007, pp. 101-104). Rather, the objection is that the two core commitments at the centre of the dual-aspect view themselves imply that a property's dispositionality is not a real, actual feature of an object. At the core of the view is a particularly thin understanding of what it is to be 'qualitative': that for something to be 'qualitative' means that it is an actual real feature of the object. So, if we claim that a property's dispositional aspect is not qualitative (as the dual-aspect view does), then we have to accept that the dispositionality of a property isn't an actual real feature of the object. Given this implausible result, the dual-aspect view should be rejected.

\section{The Future of Powerful Qualities?}

There is a growing desire in the metaphysics of properties for a view that effectively steers between the twin extremes of categoricalism and pure powers. This is the impetus for the powerful qualities view. Giannotti's paper represents an important and interesting attempt to develop this middle path. If what I have argued here is correct, he is unsuccessful.

There are two challenges that a powerful qualities theorist must meet, if they wish to distinguish their view from the pure powers view, whilst retaining a plausible metaphysics of properties. The first challenge is to develop a robust and substantive notion of a 'quality'. This is required, as the thin notion employed by Giannotti (as

\footnotetext{
6 Thanks to an anonymous referee for pushing me on this.
} 
well as others) generates a lot of the worries that the present paper has uncovered. This robust notion of 'quality' must be able to distinguish the powerful qualities view from the pure powers view, and it must be independently plausible that properties really are qualitative in this sense. The second challenge is to develop a view of the metaphysical relation between qualities (so understood) and the dispositional nature of a property. As I have argued at length, identity simply cannot be the correct relation, because if we identify a quality with a dispositionality, it will always follow that dispositionality is sufficient to fix the identity of the quality, and the regress argument will apply to the view. If these two challenges are met, then we will have found our middle path. Whether they can be met is, at present, anything but clear.

Acknowledgements Thanks to three anonymous referees for their comments, which improved the paper greatly.

Open Access This article is licensed under a Creative Commons Attribution 4.0 International License, which permits use, sharing, adaptation, distribution and reproduction in any medium or format, as long as you give appropriate credit to the original author(s) and the source, provide a link to the Creative Commons licence, and indicate if changes were made. The images or other third party material in this article are included in the article's Creative Commons licence, unless indicated otherwise in a credit line to the material. If material is not included in the article's Creative Commons licence and your intended use is not permitted by statutory regulation or exceeds the permitted use, you will need to obtain permission directly from the copyright holder. To view a copy of this licence, visit http://creativecommons.org/licen ses/by/4.0/.

\section{References}

Armstrong, D. (1997). A world of states of affairs. Cambridge: CUP.

Bird, A. (2007). Nature's metaphysics. New York: OUP.

Giannotti, J. (forthcoming). The identity theory of powers revised. Erkenntnis. https://doi.org/10.1007/ s10670-019-00122-5.

Heil, J. (2003). From an ontological point of view. Oxford: OUP.

Heil, J. (2012). The universe as we find it. Oxford: OUP.

Jacobs, J. (2011). Powerful qualities, not pure powers. The Monist, 94, 81-102.

Lewis, D. (1983). New work for a theory of universals. The Australasian Journal of Philosophy, 61, 343-377.

Lowe, E. J. (2006). The four category ontology. New York: OUP.

Martin, C. B. (2008). The mind in nature. Oxford: OUP.

Mørch, H. (2018). The evolutionary argument for phenomenal powers. Philosophical Perspectives, 31, $293-316$.

Taylor, H. (2013). In defence of powerful qualities. Metaphysica, 14, 93-107.

Taylor, H. (2017). Powerful qualities, phenomenal concepts and the new challenge to physicalism. The Australasian Journal of Philosophy, 69, 53-66.

Taylor, H. (2018). Powerful qualities and pure powers. Philosophical Studies, 175, 1423-1440.

Publisher's Note Springer Nature remains neutral with regard to jurisdictional claims in published maps and institutional affiliations. 